\title{
Evidence for electrical spin tunnel injection into silicon
}

\author{
C. L. Dennis ${ }^{\text {a) }}$ and J. F. Gregg \\ Clarendon Laboratory, University of Oxford, Parks Road, Oxford OX1 3PU, United Kingdom \\ G. J. Ensell \\ Department of Electronics and Computer Science, University of Southampton, Southampton SO17 1BJ, \\ United Kingdom \\ S. M. Thompson \\ Department of Physics, University of York, York YO10 5DD, United Kingdom
}

(Received 6 February 2006; accepted 16 June 2006; published online 30 August 2006)

Electrical spin injection into silicon was studied in a ferromagnet/insulator/silicon/insulator/ ferromagnet structure, where the insulator is $\mathrm{Si}_{3} \mathrm{~N}_{4} \cdot \mathrm{Si}_{3} \mathrm{~N}_{4}$ barriers conduct by hopping conduction at low voltages, but switch to Fowler-Nordheim tunneling at high voltages. In the Fowler-Nordheim tunneling regime a magnetic field dependence of the output current consistent with spin dependent transport through the silicon is observed; in the hopping conduction regime reduced magnetic field dependence of the output current is observed. This voltage dependence of the magnetic sensitivity strongly supports the existence of spin injection into silicon. After correction for Lorentz magnetoresistance, the magnitude of this signal is $4.1 \% \pm 0.5 \%(12 \% \pm 5 \%)$ for $p$-type ( $n$-type) Si.

(C) 2006 American Institute of Physics. [DOI: 10.1063/1.2229870]

\section{INTRODUCTION}

One of the leading contemporary challenges in spintronics is achieving spin dependent transport through silicon as a prelude to making Si-based spintronic devices. Semiconductors in the III-V family have readily yielded their secrets and transported spin polarized carriers. Optical pumping experiments led the way by demonstrating the feasibility of spin transport through GaAs and InAs and measuring the associated spin lifetimes. ${ }^{1,2}$ Spin injection demonstrations ${ }^{3-5}$ followed in which a magnetic semiconductor (or a ferromagnetic metal through a Schottky barrier or an $\mathrm{Al}_{2} \mathrm{O}_{3}$ tunnel barrier) injected spins into a III-V light emitting diode structure: the polarization of the light emitted very convincingly tracked both the magnetic hysteresis and the magnetization versus temperature characteristics of the detector, thereby confirming successful spin injection.

However, in contrast to the III-V spin transport success story, spin transport through $\mathrm{Si}$, the world's leading commercial semiconductor, remains intractable. Its indirect band gap precludes optical generation/detection of spin polarized carriers. Electrical injection is thus the only route available for studying Si's spin transport capabilities. However, to date, conclusive attempts to inject spins into $\mathrm{Si}$ have proved elusive.

In this paper, we present experimental data in which the magnetotransport effects, though still small, point encouragingly towards the implementation of spin transport through $\mathrm{Si}$. This experimental work uses three approaches to distinguish between true spin effects and magnetotransport artifacts. First, we employ a vertical structure ${ }^{6}$ whose geometry suppresses the usual transport artifacts; second, we avoid im-

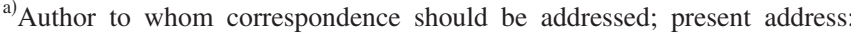
NIST, 100 Bureau Drive, Gaithersburg, MD 20899-8552; electronic mail: cindi.dennis@nist.gov
}

pedance mismatch problems ${ }^{7}$ by using spin tunnel injection ${ }^{8}$ into the silicon; third, we exploit the two conduction mechanisms in our spin injection barriers, each of which dominates in a different voltage range and one of which permits spin injection while the other does not. The latter is used to distinguish genuine spin effects from any artifacts that remain.

\section{STRUCTURE AND FABRICATION}

The device structure is shown in Fig. 1. Charge carriers are injected into a $2.2 \mu \mathrm{m}$ thick $\mathrm{Si}$ layer from a ferromagnet via a tunnel barrier by applying a voltage between the ferromagnet and the Si. This spin polarized current traverses the $\mathrm{Si}$ (by diffusion and drift) to a second tunnel barrier separating the $\mathrm{Si}$ from another ferromagnet which is back biased in order to extract carriers. The existence of a spin polarized current in the Si is indicated by the output current dependence on the relative orientation of the two ferromagnets, whose magnetizations may be differentially switched. [Since most of the spin scattering mechanisms that exist in GaAs do

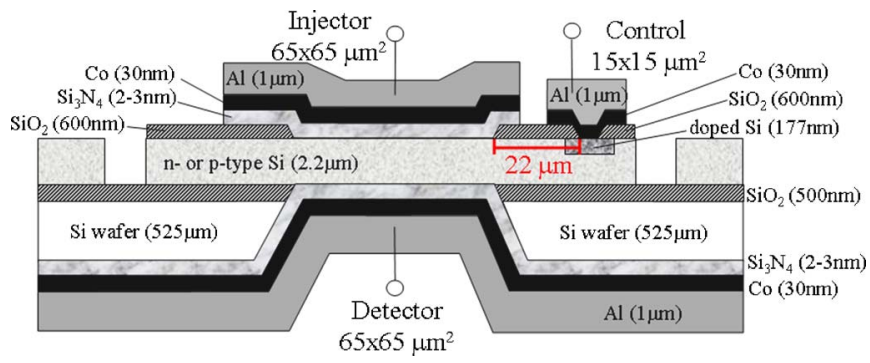

FIG. 1. (Color online) Structural schematic of the fabricated silicon device. The injector-detector separation is $2.2 \mu \mathrm{m}$ and the injector-control separation is $22 \mu \mathrm{m}$. Electrons enter from the injector and exit through the detector. The control contact determines the voltage of the silicon and therefore the relative applied voltage across the injector and detector tunnel barriers. At $I_{\text {control }}=-0.6 \mu \mathrm{A}$, the voltage drop across each of the barriers is approximately equal. 


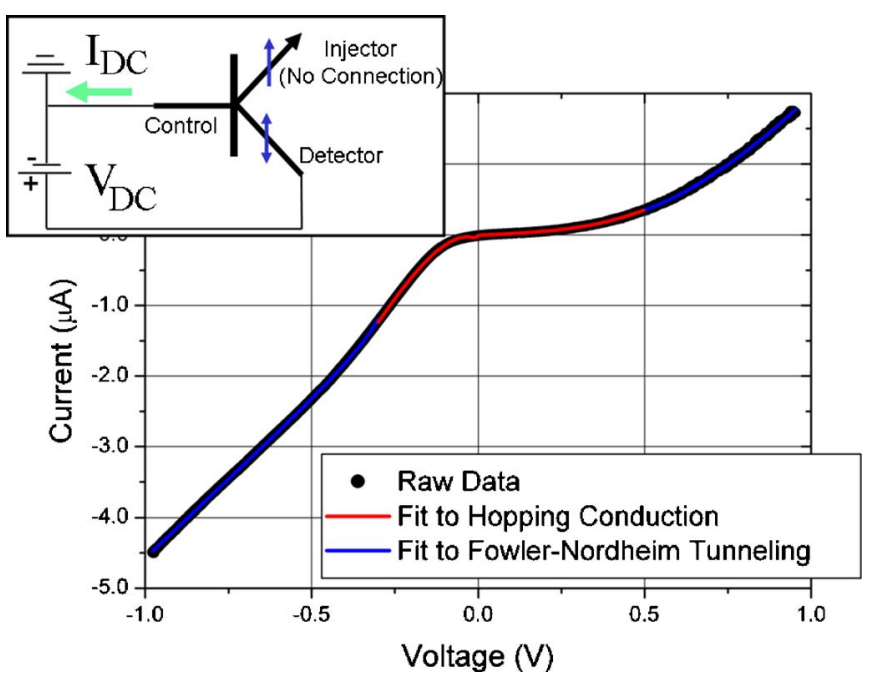

FIG. 2. (Color online) Typical example of the detector-control characteristics with the injector contact floating and the control at ground for the $p$-type Si. The dots are measured data points and the solid red and blue lines are fits to Eqs. (1) and (2), respectively, according to the region the data are in. (Inset shows the circuit diagram for these measurements where the single blue arrow indicates the "fixed" magnetic layer and the double-headed blue arrow indicates the "free" magnetic layer.)

not exist in $\mathrm{Si}^{9}{ }^{9}$ and the spin diffusion length in GaAs exceeds $100 \mu \mathrm{m}$, a spin diffusion length in $\mathrm{Si}$ of at least $2.2 \mu \mathrm{m}$ - two orders of magnitude smaller-is not unreasonable. Furthermore, electron spin resonance (ESR) work $^{10}$ from the 1950s suggests long spin lifetimes in $\mathrm{Si}$.]

The samples were fabricated using standard photolithography on $p$-type and $n$-type silicon-on-insulator (SOI) wafers with a doping of $(4-7.8) \times 10^{14} \mathrm{~cm}^{-3}$ for the $p$-type wafer and $(1.3-2.6) \times 10^{14} \mathrm{~cm}^{-3}$ for the $n$-type wafer and a resistivity of $17-33 \Omega \mathrm{cm}$ at $300 \mathrm{~K}$. (The results for the $n$ - and $p$-type $\mathrm{Si}$ are similar; due to space considerations, only the $p$-type results are shown.) The injector and detector contacts are metal-insulator-semiconductor junctions where the insulator is $\mathrm{Si}_{3} \mathrm{~N}_{4}$ and the control contact is an Ohmic metalsemiconductor junction (achieved by doping the $\mathrm{Si}$ to $10^{20} \mathrm{~cm}^{-3}$ ). For ease of fabrication, all three contacts have a thin film of cobalt, but the magnetic nature of the control contact is of no consequence as the control contact merely determines the relative applied bias across each tunnel barrier.

\section{TUNNEL BARRIER CHARACTERIZATION}

Typical room temperature $I-V$ characteristics ${ }^{11}$ of the control-detector barrier with a floating injector are shown in Fig. 2. (The results are similar for the control-injector barrier.) No single conduction mechanism across the barrier can account for the behavior over the whole voltage range. At low bias voltages, the functional form ${ }^{12}$ of the $I-V$ curves is given by

$$
I=A e^{B|V|^{-1 / 4}}+C V+D,
$$

where $A$ and $D$ have units of amperes, $B$ has units of $\mathrm{V}^{1 / 4}$, and $C$ is an admittance with units of mho. (The linear term accounts for parasitic currents.) The form of this curve implies that the dominant conduction mechanism across the barrier in this regime is Mott's variable range hopping conduction (HC), ${ }^{13,14}$ where the barrier is comprised of the $\mathrm{Si}_{3} \mathrm{~N}_{4}$ low transmission barrier and a Schottky barrier in the $\mathrm{Si}^{15}$ This is in agreement with previous work ${ }^{16-18}$ which found that $\mathrm{Si}_{3} \mathrm{~N}_{4}$ low transmission barriers conduct primarily by electron hopping near the Fermi level due to defects or dangling bonds.

At voltages greater than $\sim|0.5| \mathrm{V}$ (as determined from numerical differentiation), it can be seen that the functional form of the data changes. This change is similar to that observed previously ${ }^{16}$ and is ascribed to Fowler-Nordheim tunneling (FNT) conduction $^{19}$ overtaking the hopping conduction. ${ }^{20}$ In our structures, this change is due to injection of the carriers above the Schottky barrier and through the $\mathrm{Si}_{3} \mathrm{~N}_{4}$ low transmission barrier only. The functional form for Fowler-Nordheim tunneling [Eq. (2)] accurately fits these data for voltages greater than $\sim|0.5| \mathrm{V}$ :

$$
I=A V^{2} e^{(B / V)}+C V+D,
$$

where $A$ has units of $\mathrm{A} / \mathrm{V}^{2}, B$ has units of volts, $C$ is an admittance with units of mho, and $D$ has units of amperes. The doping type and concentration of the $\mathrm{Si}$ influence only the detailed form of the $I-V$ characteristics.

It is well established ${ }^{21}$ that hopping conduction destroys the spin polarization of carriers due to their extended time ${ }^{22}$ at each localized state for an average time that generally exceeds their spin lifetime. Therefore, little magnetic sensitivity of spin dependent origin should be exhibited by the device in the regime where hopping conduction dominates. Later, we use this observation as a means to help separate genuine spin injection effects in the device from the multitude of spurious magnetic artifacts (anisotropic magnetoresistance, Lorentz magnetoresistance, Hall effect, etc.) that plague these types of magnetotransport measurements.

\section{SPIN INJECTION EXPERIMENT}

The three-terminal $I-V$ characteristics with fixed control current, presented in Figs. 3 and 4, were measured at room temperature with a magnetic field applied in the plane of the device and perpendicular to the current direction. Owing to the substantial additional noise associated with magnetic field sweeping (due to magnetocaloric effects combined with the temperature dependence of silicon), the data were measured by sweeping the voltage/current characteristics at a selection of fixed magnetic fields. For the data presented in Figs. 3 and 4, these fixed field values were established by approaching from the negative magnetic field direction.

Due to shape anisotropy, the magnetic moments of the injector and detector pads are parallel for applied magnetic fields lower than $+2.4 \mathrm{kA} / \mathrm{m}(+30 \mathrm{Oe})$ and greater than $+9.2 \mathrm{kA} / \mathrm{m}(+115 \mathrm{Oe})$ and antiparallel for positive applied magnetic fields between $2.4 \mathrm{kA} / \mathrm{m}(30 \mathrm{Oe})$ and $9.2 \mathrm{kA} / \mathrm{m}$ $(115 \mathrm{Oe})$, as shown by the blue triangles in Fig. 3. There are three significant results from these measurements. First, the $I-V$ characteristics are a function of applied magnetic field. Second (disregarding Lorentz magnetoresistance, see below), little magnetic sensitivity is observed for voltages below the onset of Fowler-Nordheim tunneling (see Fig. 3). Third, the 


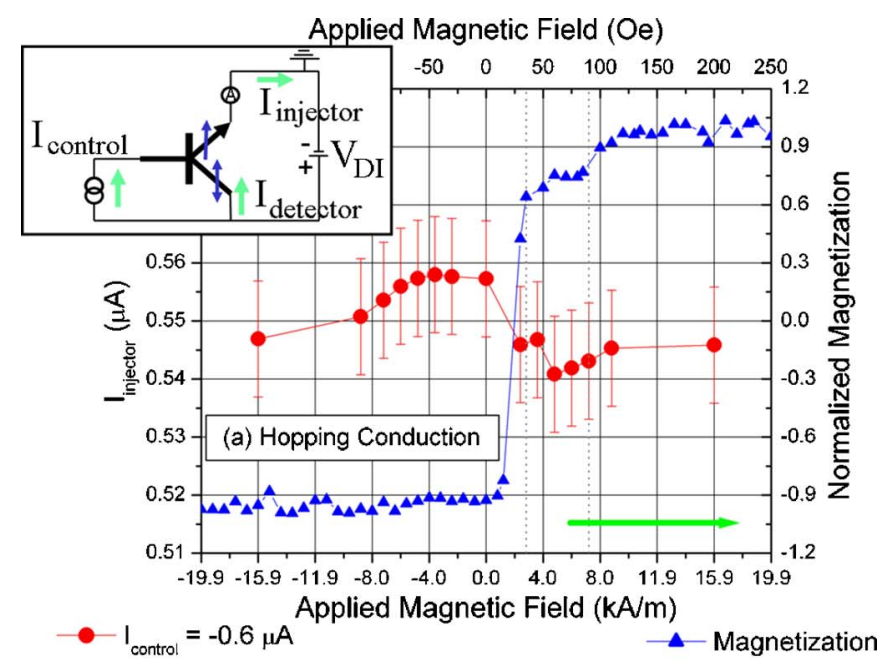

Applied Magnetic Field (Oe)

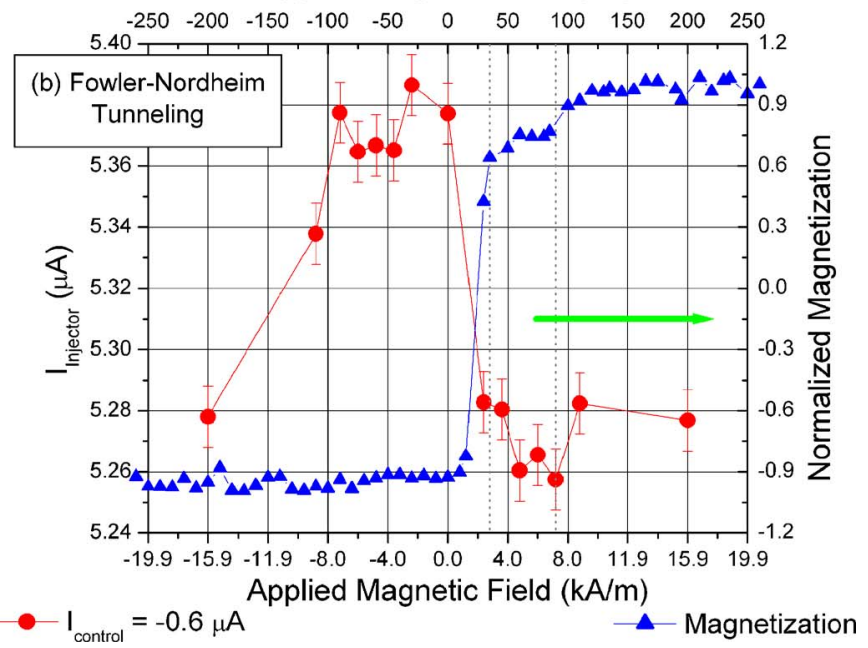

FIG. 3. (Color online) The raw injector current (red dots) as a function of applied magnetic field for the $p$-type $\mathrm{Si}$ in (top) the dominantly hopping conduction regime at $V_{\mathrm{ID}}=0.15 \mathrm{~V}$ and $I_{\text {control }}=-0.6 \mu \mathrm{A}$ and (bottom) the dominantly Fowler-Nordheim tunneling regime at $V_{\mathrm{ID}}=1 \mathrm{~V}$ and $I_{\text {control }}=$ $-0.6 \mu \mathrm{A}$. Half of a hysteresis loop as measured on a VSM is shown by the blue triangles. The green arrow indicates the direction of the magnetic field sweep of the measurements, following saturation at fields $<-80 \mathrm{kA} / \mathrm{m}$ $(-1 \mathrm{kOe})$ and the dotted vertical lines show the region of antiparallel alignment. [Inset for (a) shows the circuit diagram for these measurements where the encircled "A" represents an ammeter. The single blue arrow indicates the "fixed" magnetic layer and the double-headed blue arrow indicates the "free" magnetic layer.]

data of Fig. 4 (which are taken at an applied voltage above $e^{23}$ the HC/FNT threshold and are corrected for Lorentz magnetoresistance) show that when the magnetic moments of the injector and detector pads are parallel, the injector current is larger than in the antiparallel configuration. The magnitude of this change is $4.1 \% \pm 0.5 \%(12 \% \pm 5 \%)$ for $p$-type (n-type) Si. Furthermore, the data in the hopping conduction regime change little after correction for Lorentz magnetoresistance (LMR).

The raw data of Fig. 3 were corrected for LMR by the following: The $I-V$ characteristics of the output current at fixed control current and voltage were measured as a function of magnetic field. These data were then fitted to a polynomial of the order of 2, and the fit was used to calculate the output current purely from LMR at each of the measured

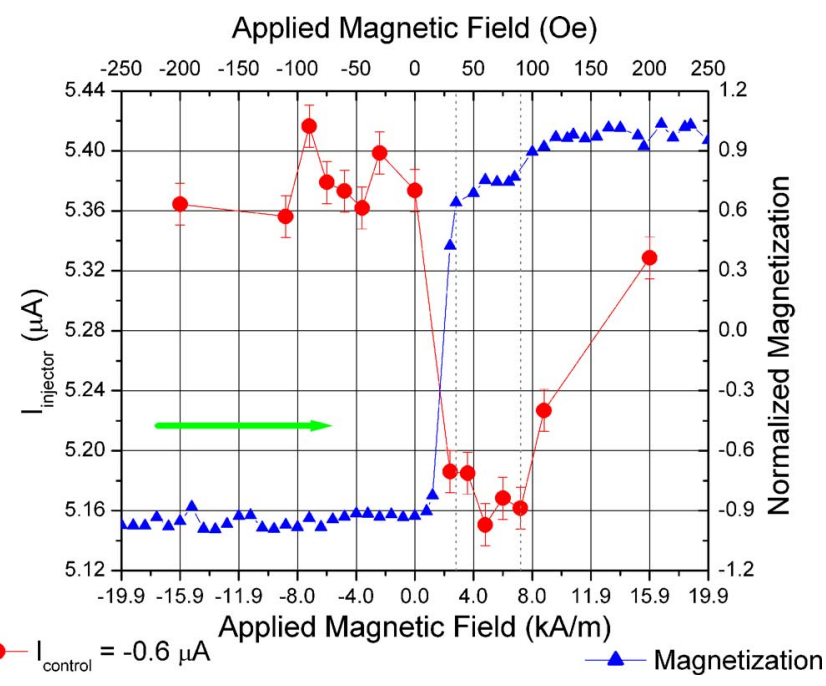

FIG. 4. (Color online) The injector current (red dots) as a function of applied magnetic field for the $p$-type $\mathrm{Si}$ after correction for LMR at $V_{\mathrm{ID}}$ $=1 \mathrm{~V}$ and $I_{\text {control }}=-0.6 \mu \mathrm{A}$ (in the dominantly Fowler-Nordheim tunneling regime). Half of a hysteresis loop as measured on a VSM is shown by the blue triangles. The green arrow indicates the direction of the magnetic field sweep of the measurements, following saturation at fields $<-80 \mathrm{kA} / \mathrm{m}$ $(-1 \mathrm{kOe})$ and the dotted vertical lines show the region of antiparallel alignment. (Note that correcting the hopping conduction data for LMR only reduces the antiparallel alignment values slightly. The changes are within the error bars and are therefore not significant.)

fields. This value was then subtracted from the raw data (with an added constant background value so that the current magnitude remains the same) to yield the processed data in Fig. 4. (It should be noted that, due to the noise associated with the magnetic field sweeping, the fit takes an average which yields only the LMR signal; this is the same regardless of the applied control current or voltage.)

\section{DISCUSSION}

To confirm the origins of the observed magnetotransport effects, we must consider not just the spin dependent tunneling effects with which we are primarily concerned but also a large family of other potential artifacts with which the spin dependent transport might be confused. These include anisotropic magnetoresistance (AMR), Lorentz magnetoresistance, and the first-order and second-order Hall effects. The magnetic fields driving these possible artifacts are twofold in origin: the actual magnetic fields that we apply to switch the electrode magnetizations (these do not exceed $80 \mathrm{kA} / \mathrm{m}$ ) and also the fringing fields at the extremities of the magnetic electrodes whose values may locally approach $1 \mathrm{~T}$ over very small volumes.

The AMR effect is typically of the order of $1 \%$. Cobalt has a nominal resistivity of $6 \mu \Omega \mathrm{cm}$ which yields a resistance of less than $450 \mathrm{n} \Omega$ for the cobalt contacts and approximately $4 \mathrm{n} \Omega$ for any AMR contribution to the magnetotransport of the device. AMR effects can thus be discounted in our measurements.

Any first-order Hall effect voltages due to the applied or fringing magnetic fields should have odd symmetry (i.e., would change sign as the applied field is reversed). From the known Hall coefficient of our SOI wafer and the device ge- 
ometry we would not expect to see a measurable Hall effect at any magnetic field in Fig. 3. That there is no such linear term in the magnetotransport is confirmed. Even more importantly, there is no significant component with odd symmetry in the signal as a function of magnetic field that follows the magnetization of the magnetic contacts. This latter observation allows us to conclude that the Hall effect due to fringing fields may also be neglected: although these fringing fields may be large over a restricted volume, they are apparently sufficiently far from the main current path not to influence the transport. This observation also enables us to put an upper limit on the effect of the fringing fields in causing both the second-order Hall effect and LMR (both of these signals are symmetric and hence potentially confusable with a spin injection signal). Consequently, we can also discount these fringe field effects as possible origins of the observed symmetric magnetotransport.

The final potential artifact is LMR due to the applied magnetic field. This is definitely present and clearly observable (data not shown). Since any LMR in the ferromagnet which is only $30 \mathrm{~nm}$ thick (compared to $2.2 \mu \mathrm{m}$ for the $\mathrm{Si}$ ) is negligibly small, the effect originates solely from the Si. Its magnitude agrees with predictions-approximately $4 \%$ $(\sim 40 \mathrm{k} \Omega)$ of the total resistance over the applied field range. This is also a symmetric (parabolic) effect and we have subtracted it from the data of Fig. 3 to yield Fig. 4 [where its primary effect is to alter the injector currents of the $H$ $= \pm 16 \mathrm{kA} / \mathrm{m}( \pm 200 \mathrm{Oe})$ data] to leave a $4 \%$ signal variation that we believe arises from spin dependent transport.

Our confidence in this result arises not only from having accounted for the potential competing artifacts but also from the correlation between the observed spin dependent effect and the voltage regime of the barriers. If spin dependent transport was absent, then the two different methods of conduction (Fowler-Nordheim tunneling and hopping conduction) should have no influence on the magnetic signal. The type of magnetic sensitivity present at low voltages should also be present at high voltages, and vice versa. In practice the onset of strong asymmetric magnetic sensitivity is voltage dependent. This is consistent with the hopping conduction substantially destroying the spin polarization of the carriers in the low bias regime; then when bias is increased and Fowler-Nordheim tunneling begins to dominate, the spin polarization is retained, rendering the current sensitive to the relative magnetic orientations of the magnetic detector and injector contact pads. Assuming this mechanism, the spin polarization can be estimated from Julliere's model ${ }^{24}$ for spin tunneling to be $1.40 \% \pm 0.08 \%$ for the $p$-type $\mathrm{Si}$. (Assuming holes are the carriers in the $n$-type $\mathrm{Si}$, the same arguments apply and yield a spin polarization of $1.09 \% \pm 0.05 \%$.) This value for the spin polarization is significantly lower than the theoretical value of $38 \%$, as well as lower than typical values in the literature. This is to be expected since hopping conduction, though no longer dominant, is still active at high barrier bias and it destroys part of the injected spin polarization.

\section{CONCLUSIONS}

In conclusion, two different conduction mechanisms with different voltage regimes were observed in the $\mathrm{Si}_{3} \mathrm{~N}_{4}$ low transmission barriers, one of which destroys spin. When combined with symmetry considerations from the device geometry, this voltage dependence of the magnetic sensitivity strongly supports the existence of spin injection into silicon.

\section{ACKNOWLEDGMENTS}

The authors thank the silicon processing team at the University of Southampton for fabricating the devices; C. V. Tiusan, M. Hehn, M. E. Flatté, R. Kirschman, and P. D. Sparks for useful discussions; and the EPSRC (GR/L68643/ 01) and the EC project SPINOSA for financial support.

${ }^{1}$ J. M. Kikkawa and D. D. Awschalom, Nature (London) 397, 139 (1999). ${ }^{2}$ T. F. Boggess, J. T. Olesberg, C. Yu, M. E. Flatté, and W. H. Lau, Appl. Phys. Lett. 77, 1333 (2000).

${ }^{3}$ R. Fiederling, M. Keim, G. Reuscher, W. Ossau, G. Schmidt, A. Waag, and L. W. Molenkamp, Nature (London) 402, 787 (1999); Y. Ohno, D. K. Young, B. Beschoten, F. Matsukura, H. Ohno, and D. D. Awschalom, ibid. 402, 790 (1999).

${ }^{4}$ V. F. Motsnyi, J. de Boeck, J. Das, W. van Roy, G. Borghs, E. Goovaerts, and V. I. Safarov, Appl. Phys. Lett. 81, 265 (2002).

${ }^{5}$ A. T. Hanbicki et al., Appl. Phys. Lett. 82, 4092 (2003).

${ }^{6}$ C. L. Dennis, C. Sirisathitkul, G. J. Ensell, J. F. Gregg, and S. M. Thompson, J. Phys. D 36, 81 (2003).

${ }^{7}$ G. Schmidt, D. Ferrand, L. W. Molenkamp, A. T. Filip, and B. J. van Wees, Phys. Rev. B 62, R4790 (2000).

${ }^{8}$ E. I. Rashba, Phys. Rev. B 62, R16267 (2000).

${ }^{9}$ D. I. Pugh, Ph.D. thesis, University of York, 2001.

${ }^{10}$ G. Feher and E. A. Gere, Phys. Rev. 114, 1245 (1959).

${ }^{11}$ Nearly all of the voltage dropped is across the interface barrier. The $\mathrm{Si}$ accounts for at most $0.5 \%$ of the voltage drop and at least $0.01 \%$ of the voltage. In effect, the resistance of the Si can be neglected entirely.

${ }^{12}$ Many functional forms were tried, all using at least four parameters, including L. I. Glazman and K. A. Matveev [Sov. Phys. JETP 67, 1276 (1988)] inelastic tunneling. However, the data either visibly did not fit (reduced $\chi^{2}>10^{-8}$ ) or yielded unphysical values.

${ }^{13}$ N. F. Mott and E. A. Davis, Electronic Processes in Non-Crystalline Materials (Oxford University Press, New York, 1979).

${ }^{14}$ Y. Xu, D. Ephron, and M. R. Beasley, Phys. Rev. B 52, 2843 (1995).

${ }^{15}$ C. L. Dennis, C. V. Tiusan, R. A. Ferreira, J. F. Gregg, G. J. Ensell, S. M. Thompson, and P. P. Freitas, J. Magn. Magn. Mater. 290-291, 1383 (2005).

${ }^{16}$ C. Chaneliere, J. L. Autran, S. Four, R. A. B. Devine, and B. Balland, J. Non-Cryst. Solids 245, 73 (1999).

${ }^{17}$ Y. Manabe and T. Mitsuyu, J. Appl. Phys. 66, 2475 (1989).

${ }^{18}$ T. Goto and T. Hirai, J. Mater. Sci. 24, 821 (1989).

${ }^{19}$ R. H. Fowler and L. W. Nordheim, Proc. R. Soc. London, Ser. A 119, 173 (1928).

${ }^{20}$ It should be noted that the work function of Co with $\mathrm{Si}$ is approximately $0.6 \mathrm{eV}$. Since conduction over the Schottky barrier would not be expected until the tunnel barrier supports roughly the same voltage (actually slightly less), this lends additional support to this interpretation of the results. Furthermore, there is a factor of 4 difference between the onset of FowlerNordheim tunneling in our devices $(0.5 \mathrm{~V})$ compared to the double structures $(2 \mathrm{~V})$ in Ref. 16. This correlates well with the thickness difference: $2 \mathrm{~nm}$ in our barriers as opposed to $8 \mathrm{~nm}$ in the double structures.

${ }^{21}$ S. Sundar Manoharan, D. Elefant, G. Reiss, and J. B. Goodenough, Appl. Phys. Lett. 72, 984 (1998).

${ }^{22}$ It is assumed that the total hopping time is much greater than the tunneling time. This is a reasonable assumption given that the thickness of the Schottky barrier exceeds approximately $500 \mathrm{~nm}$.

${ }^{23} I_{\text {control }}$ was held constant at $-0.6 \mu \mathrm{A}$ to engineer an approximately equal split of $V_{\mathrm{DI}}$ between the detector and injector tunnel barriers. Therefore, the regime classification used for Fig. 2 can be used here since the voltage regimes are similar for the detector and the injector tunnel barriers.

${ }^{24}$ M. Julliere, Phys. Lett. 54, 225 (1975). 